\title{
Brief Wisdom Screening Scale: Multi-sample factor analysis and validation
}

\author{
Thomas Anderson
}

What is wisdom and how can we measure it in a questionnaire? Such debates pervade the literature and different theories result in different tools. One measure differentiates itself as a theory-independent, empirically constructed scale: The Brief Wisdom Screening Scale (BWSS). Scant research focuses on validation or subscale analysis of the BWSS thus we performed four studies. Study $1(n=389)$ applies exploratory factor analysis (EFA), uncovering a twofactor structure with "Poise" relating to an internalized, equanimous component of wisdom and "Attunement" relating to the learning-from-life orientation of wisdom. Study 2-4 ( $n=331)$ applied confirmatory factor analysis in a new pre-registered sample. Two-factor models provide superior fit and exploratory correlational analyses provide evidence for construct validity. Attempting to replicate the EFA, however, raised additional questions. Amid disagreement about wisdom definitions and measurement, the empirically constructed BWSS may provide data-driven insights to inform theoretical perspectives from the bottom up.

\section{Intelligence is knowing tomatoes are a fruit; Wisdom is knowing not to put tomatoes in a fruit salad.}

"Wisdom" is one of the more challenging concepts to define and measure (Baltes et al., 2002). The Oxford English Dictionary defines wisdom as the "capacity of judging rightly in matters relating to life and conduct; soundness of judgement in the choice of means and ends" ('Wisdom, n.', n.d.). This capacity exists on a spectrum of expertise and the practised experience of coming to appropriate conclusions and behaviourally implementing such conclusions has resulted in a conceptualization of wisdom as "expertise in the conduct of life" (Baltes et al., 2002). Researchers continue to disagree about the exact origin and application of this expertise, including whether wisdom is a skill, attitude, or personality trait, and discussions continue regarding whether wisdom is primarily concerned with personal reflection, social reasoning, advice-giving, or still other domains (Glück, 2018; Igor Grossmann et al., submitted).

With debates about definitions continuing to persist, it is no wonder that another enduring issue in the study of wisdom is the question of how to measure wisdom. There are two main methods for measuring wisdom used in modern research: task-based assessments of wisdom and self-report measures of wisdom (Glück, 2018); there is no commonly used informant-based measure of wisdom. For research in which wisdom is the primary construct of interest, task-based measures that operate on interviews or open-response questions, each of which must be subjectively rated by trained judges, may be appropriate, but for research where wisdom is one of many variables of interest, selfreport measures are more practicable. A number of theorybased self-report measures of wisdom have been created, typically exemplifying the scale-author's particular definition of wisdom (see (Glück, 2018; Glück et al., 2013) for reviews of these measures). There is one scale, however, that was developed from a purely empirical approach: the Brief Wisdom Screening Scale (BWSS, (Glück et al., 2013)).

Glück et al. (Glück et al., 2013) developed the BWSS by identifying the items in three different self-report measures that had the highest correlations with a common general wisdom factor across several measures of wisdom; this method was cross-validated by splitting the sample in two. The empirically extracted wisdom self-report factor was able to explain considerable variance (sample 1: $82.9 \%$, sample 2: $65.0 \%$ ), correlated very strongly with the primary wisdom factor score (sample 1: $r=0.90$, sample 2: $r=0.92$ ), and presented with an excellent Cronbach's alpha (both samples: 0.87). While this scale appears face-valid and its empirical orientation circumvents some of the definitional debates, this scale has been underused, perhaps due to a lack of validation studies. The present set of four studies aims to validate the BWSS.

\section{The Present Study}

We include data from 4 studies (total $\mathrm{N}=720$ ) with
Department of Psychology, University of Toronto, Toronto, ON, Canada.

*Corresponding Author e: metathomas.anderson@ mail.utoronto.ca 
the intent to validate the BWSS. Data from an existing study ( $\mathrm{n}=389$ ) were subjected to Confirmatory Factor Analysis (CFA), which failed, then Exploratory Factor Analysis (EFA), resulting in a two-factor structure and the recommendation to drop one item from the scoring of the scale. We uploaded the two-factor results to the Open Science Framework (OSF) and pre-registered a CFA to validate the structure in a new study (Anderson, 2018a). This new study had lower than anticipated recruitment (aim: 200-400, actual $n=65$ ) so we added the BWSS as an additional measure to two additional studies, supplementing data collection to reach the registered sample size goal (explained further below).

\section{Study 1}

\section{Study 1 Methods}

Study 1 was a pre-registered study on microdosing psychedelics that was not originally intended to be used for BWSS validation (Anderson, Petranker, Rosenbaum, et al., 2019). The BWSS data from that survey study were subjected to EFA, which inspired the registration and subsequent intent of validating a two-factor structure for the BWSS. A full report of (Anderson, Petranker, Rosenbaum, et al., 2019)'s methods and primary findings are available in the original article; an abridged version as pertains to the BWSS data is reported here.

\section{Study 1 Participants}

BWSS scores were obtained from 389 participants, primarily recruited via "reddit" (Reddit Inc, San Francisco, CA, USA). Participation was not remunerated. This English-language survey was available internationally and respondents originated in 29 different countries, though countries from the Anglo cultural cluster made up $>70 \%$ of the sample. Middle-class, white, male, heterosexual adults made up the majority of the sample (mean age: 27.58, SD: 9.22 , range $16-70 ; 313$ male). Due to the nature of the original study, nearly all respondents had experience with psychedelics.

\section{Study 1 Design and BWSS}

Participants consented, then engaged questionnaires and online tasks. Scales, including the BWSS (Glück et al., 2013), were rated on continuous 0-100 slider-scales with nominal descriptors at 0 ("Disagree Strongly") and
100 (“Agree Strongly”). Question-order was randomized. Reliability was not decreased by the scale transformation $(\alpha=0.85)$. Microdosers were found to have higher wisdom than non-microdosers (Anderson, Petranker, Rosenbaum, et al., 2019).

\section{Study 1 Other Questionnaires}

Participants completed other questionnaires, which we explore as exploratory correlations (see Exploratory Analysis for Construct Validation). Of relevance, participants completed a mood-board [cite OSF], the Dysfunctional Attitudes Scale (DAS, (de Graaf et al., 2009)), the Openness subscale of the Big Five Personality Inventory 2 (BFI-2, (Soto \& John, 2017)), and the Emotional Negativity subscale of the BFI-2 (also called "Neuroticism").

\section{Study 1 Results - Factor Analysis}

The R Language (R Core Team, 2014) was used for statistical analyses using the psych and lavaan packages for modelling (Revelle, 2020; Rosseel et al., 2018) and the semPlot and ggplot2 packages for visualization (Epskamp et al., 2019; Wickham, 2016). The same packages are used for Studies 2-4.

BWSS responses were subjected to a confirmatory factor analysis using one latent factor. Noting the poor fit indices of that one-factor model (Table 1), we subjected the data to exploratory factor analysis. The BWSS had a meritorious Kaiser-Meyer-Olkin factor adequacy of 0.89 thus the eigenvalues and scree plot were examined (Figure 1), revealing that a two-factor solution may be more appropriate.

As the BWSS was constructed to model a general factor of wisdom, the two-factor fit was expected to have correlating factors thus oblique rotations were used. Seven oblique rotation methods were computed and in every case (other than "simplimax") the same items loaded (>0.3) onto the same factors, and in every case one item did not load onto either factor ("There are some people I know I would never like", reverse-coded). The Promax rotation is reported here (see Appendix) and all rotations are provided in Supplemental Materials.

\section{Study 1 Interim Discussion}

The BWSS is an empirically generated scale based on correlations with a general factor of wisdom (Glück et al., 2013); a two-factor model fits the data from our sample.
Table 1

Goodness-of-Fit Indices for CFA and EFA in all studies

\begin{tabular}{|c|c|c|c|c|}
\hline Sample & BWSS & CFI | TLI & RMSEA & BIC \\
\hline Study 1 & One-Factor & $0.700 \mid 0.667$ & 0.093 & 75501.171 \\
\hline Study 1 & One-Factor rev & $0.707 \mid 0.672$ & 0.097 & 71666.292 \\
\hline Study 1 & Two-Factor & $0.833 \mid 0.812$ & 0.073 & 71405.406 \\
\hline Studies 2-4 & One-Factor & $0.763 \mid 0.737$ & 0.081 & 65012.057 \\
\hline Studies 2-4 & One-Factor rev & $0.775 \mid 0.749$ & 0.083 & 61758.976 \\
\hline Studies 2-4 & Two-Factor & $0.801 \mid 0.777$ & 0.078 & 61718.591 \\
\hline \multicolumn{5}{|c|}{$\begin{array}{l}\text { Note: Studies } 2-4 \text { are reported grouped together; for a breakdown by separate studies, see Supplemental } \\
\text { Table 1. "One-Factor" includes all items; "One-Factor rev" drops an ill-fitting item (\#20, discussed further } \\
\text { below). The Two-Factor model had superior fit. No model resulted in CFI/TLI > 0.85; all models resulted in } \\
\text { RMSEA under 0.10. CFI: Comparative Fit Index; TLI: Tucker Lewis Index; RMSEA: Root Mean Square Error } \\
\text { of Approximation; BIC: Bayesian information criterion }\end{array}$} \\
\hline
\end{tabular}




\section{Scree Plot For Study 1}

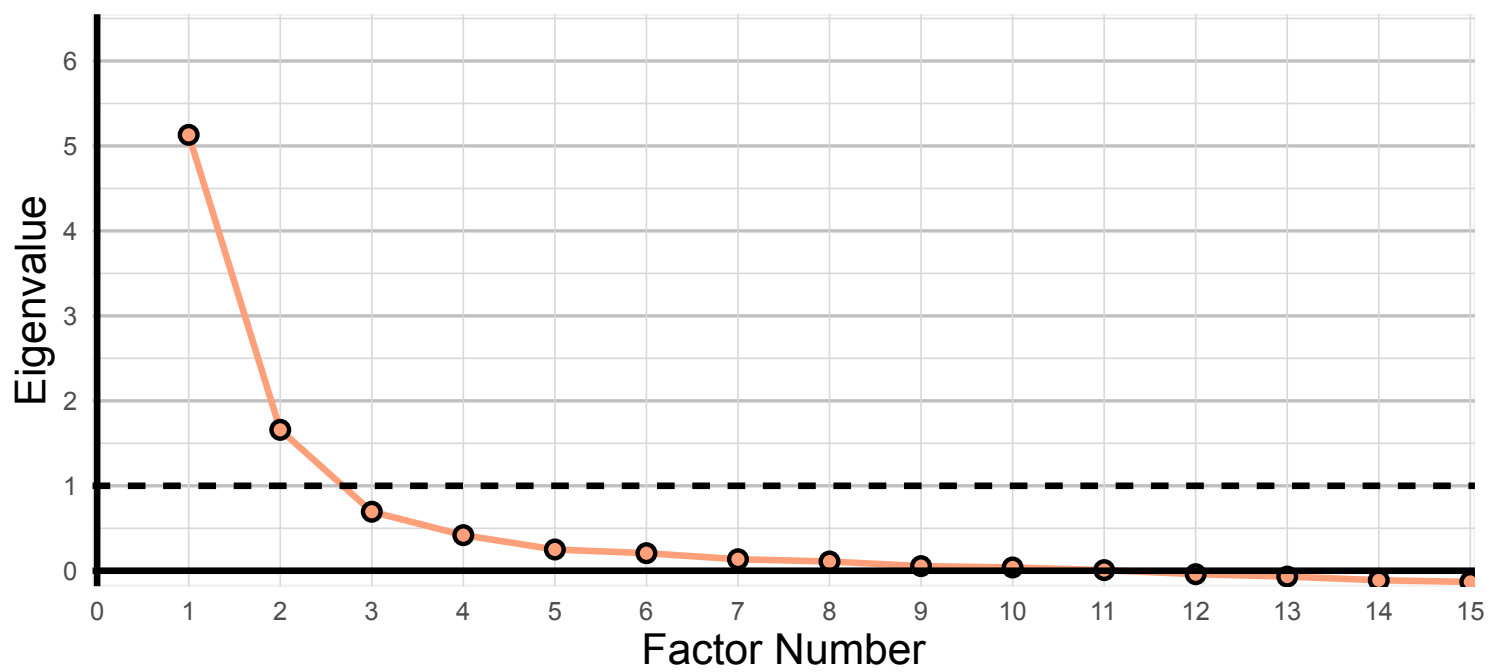

Study $\quad 0$ Microdose Study (1)

Examining the items that significantly load on each factor (see Appendix) reveals two conceptually distinct groups of related items. The two factors uncovered here - which we name "Poise" and "Attunement" - align well with other classes of wisdom measures discussed in Glück et al. (2013): "Personal", "Other-related", and "General" wisdom.

The first factor includes items tapping stability of mind, acceptance, humour, and an independence from the vicissitudes of life. This factor - dubbed Poise - reflects the internal component of wisdom as one's orientation toward individual experience and could be considered amid concepts such as psychological integration, individuation, or self-actualization, and amid philosophical concepts such as Buddhist equanimity, Stoic ataraxia, or a generalized "inner peace". In this sense, Poise reflects stability.

The second factor includes items tapping one's propensity to have and learn from varied experiences, including learning from relationships with diverse others and their belief systems, awareness of and understanding of one's own emotions and the emotions of others, and intentionally considering varied perspectives and one's place in a "greater whole". This factor - dubbed Attunement - reflects the component of wisdom that concerns the distillation of experiences into life lessons through one's engagement with others and the world. In this sense, Attunement reflects change and openness to change.

Across the various rotations, up to four of the items loaded on both factors: "I am able to integrate the different aspects of my life", "I can accept the impermanence of things", "I have a good sense of humor about myself", and "I always try to look at all sides of a problem". These items may reflect common core elements of wisdom gained across both intrapersonal and interpersonal experiences.

One item (item 20: "There are some people I know I would never like", reverse-coded) loaded on neither factor. This item may have been intended to tap prejudice (lower wisdom), but may simultaneously tap genuine insights into personality and social conflict learned through experience (higher wisdom). Perhaps, in certain cases, having recognized the traits of those with whom one most and least fruitfully associates reflects varied self-knowledge and interpersonal experiences, dividing the item and reducing its utility.

Anticipating the need for additional studies to investigate this two-factor structure for the BWSS we registered Study 2, which measured BWSS in the context of meditation practices.

\section{Studies 2-4}

\section{Studies 2-4 Methods}

Study 2 was a pre-registered study intended to apply CFA to the BWSS to validate the two-factor structure uncovered in Study 1. We registered our intent to collect a sample of 200-400 participants in one longitudinal online study, the Meditation and Practice (MAP) study (see below). Unfortunately, participant recruitment was much lower than expected $(n=92)$. In order to maintain the primary goal described in the Study 2 Sample Size Rationale of collecting a sufficiently large sample to test the CFA, we administered the BWSS in two additional studies otherwise unrelated to Study 2. BWSS data from Study 2 were not analysed prior to the decision to collect more data. Studies 3-4 (elaborated below) aimed to collect equivalent sample sizes, but data collection for Study 4 concluded early due to COVID-19-related closures. A total sample of 331 was collected across the three CFA data-sets: Study 2 [MAP] (n = 92), Study 3 [MCT Long] $(\mathrm{n}=194)$, and Study 4 [MCT $\mathrm{B} \& \mathrm{~W}](\mathrm{n}=45)$.

\section{Study 2 Participants}

Community members were recruited online in exchange for financial payment at a rate of $10 \mathrm{CAD} /$ hour. Participants were meditating adults (mean age: 31.16 , SD:
Figure 1

Screen plot of Sample

1 BWSS Factor

Analysis. 
10.37, range 18.17-64.42; 37 male). Recruitment took place between January and July 2019; this deviated from the pre-registration in that recruitment was to stop April 2019, but was extended due to low participation.

\section{Study 2 Procedures}

Study 2 Meditation and Practice (MAP)

Contemplative practices are thought to promote personal mental health and interpersonal social functioning, but there are almost no longitudinal studies that demonstrate relations between weekly contemplative practice and personal or social functioning. Study 2's primary goal was to measure associations between contemplative practice, subjective well-being, and social function on a weekly basis over 6 months; participants completed the BWSS at baseline after reporting demographics and their contemplative background and before completing other questionnaires.

\section{Study 2 Data Exclusion}

As registered, participants were removed for "troll" responses, which included a number of "bots", i.e. automated survey responses $(n=46)$. Bots were qualitatively identified by sanity-checking responses. In particular, this study requested the respondent's name in addition to three informants; if the informant names and respondent names were identical, the response was coded as a bot and removed. Additionally, participants reported the name of their meditation practice and were removed if they reported inappropriate responses (e.g. "Living on the Moon", "Winning Cupid's Award"); all but 5 of these inappropriate responses were from respondents previously identified as bots. Participants were also dropped if they did not complete all questions in the BWSS $(\mathrm{n}=27)$.

\section{Study 2 Other Questionnaires}

Participants completed many questionnaires, in addition to the BWSS, that can be explored to demonstrate construct validity. After the BWSS, participants completed the following: Patient Health Questionnaire - Somatic, Anxiety, and Depressive Symptoms (PHQ-SADS, (Kroenke et al., 2010)), Ten-Item Personality Inventory (TIPI, (Gosling et al., 2003)), Five-Factor Mindfulness Questionnaire Short Form (FFMQ-SF, (Bohlmeijer et al., 2011)), Acceptance and Action Questionnaire II (AAQ-II, (Bond et al., 2011)), Emotion Regulation Questionnaire (ERQ, (Gross \& John, 2003)), Experiences Questionnaire (EQ, (Fresco et al., 2007)), Rumination Reflection Questionnaire (RRQ, (Trapnell \& Campbell, 1999)), and a number of other scales not explored here (Farb et al., 2018).

\section{Studies 3-4 Participants}

Unlike the community samples in Studies 1 and 2, participants in Studies 3-4 were undergraduate students (Study 3: $\mathrm{n}=194$; mean age: 19.42, SD: 2.09, range 17-33.5; 58 male; Study 4: $\mathrm{n}=45$; mean age: 19.74, SD: 1.69 , range $18.08-26.17$; 7 male). Studies 3-4 took place at the University of Toronto, Mississauga campus, between May 2019 and April 2020 and participants were undergraduates participating in exchange for course-credit. Data was collected under informed consent in accordance with the Declaration of Helsinki under the university's Social Sciences, Humanities, and Education Research Ethics Board.

\section{Studies 3-4 Procedures}

Studies 3-4 Metronome Counting Task (MCT)

Prior to completing the BWSS, participants were engaged in a behavioural task, the Metronome Counting Task (MCT, [cite MCT paper]), validation of which was the primary purpose of the study and procedures for which are described fully in the primary MCT paper. Briefly, participants in the MCT tapped along to a steady audio cue while counting. Participants completed four 10 minute blocks with self-paced breaks between. In Study 3 (MCT Long), feedback was colour-coded to indicate performance. In Study 4 (MCT B\&W), feedback was not colour-coded. The studies were otherwise identical.

\section{Studies 3-4 Data Exclusion}

In the MCT studies, participants could be excluded if they indicated they disengaged from the MCT portion of the experiment or for indicating they did not understand the MCT instructions. Since the MCT portions are not of interest when considering validation of the BWSS, such participants were not excluded. Participants were dropped if they did not complete all questions in the BWSS (Study 3: $\mathrm{n}=2$; Study $4: \mathrm{n}=0$ ).

\section{Studies 3-4 Other Questionnaires}

Participants completed a variety of other questionnaires in addition to the BWSS, which will be explored for construct validation. Participants completed a mood-board [cite OSF?], the Positive and Negative Affect Schedule (PANAS, (Watson et al., 1988)), the Attention Related Cognitive Errors scale (ARCES, (Cheyne et al., 2006)), and the Mind Excessively Wandering Scale (MEWS, (Mowlem et al., 2019))

\section{Studies 2-4 Results - Confirmatory Factor Analysis}

BWSS responses were obtained from 331 participants and were subjected to a CFA using the factor structure found in Study 1. As registered, we report CFI, TLI, RMSEA, and BIC for fit indices and model comparisons (Table 1). Consistent with predictions, the two-factor model was the superior fit.

\section{Studies 2-4 Results - Replication Attempt - Exploratory Factor Analysis}

In an attempt to replicate the specific factor structure from Study 1, EFA was also applied to Study 2-4. While performing an EFA after a CFA is unconventional, the results are telling, highlighting the need for caution when generalizing. The screen plots for the combined sample and three individual samples are presented in Figure 2. As can be seen, a two-factor model is again suggested. The same seven oblique rotation methods were computed (see Supplemental Materials). Counter to expectations, the item loadings were different from Study 1. Most items loaded (>0.3) onto one factor, two reverse-scored items loaded onto a second factor, 


\section{Scree Plot By Study}

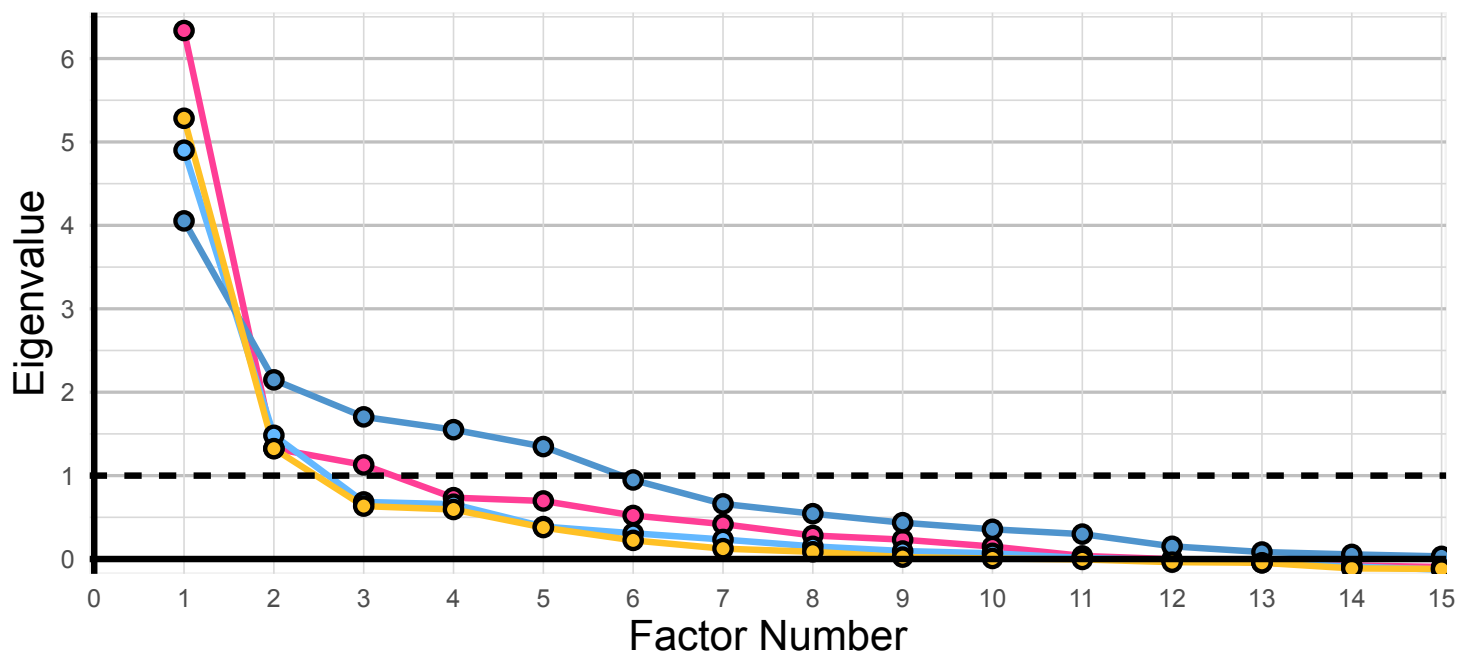

Study - $\mathrm{O}$ MAP Study (2) $\mathrm{O}$ MCT Long (3) $\mathrm{O}$ MCT B\&W (4)
Figure 2

Screen plot of Samples 2-4 BWSS

Factor Analysis. one item loaded positively on both factors, and one item loaded positively on factor 1 and negatively on factor 2 . Consistent with Study 1, however, item 20 ("There are some people I know I would never like.”) did not load onto either factor. Another notable difference was the proportion of variance explained by the factors. While cumulative variance was similar (Study 1: 33\%; Study 2-4: 32\%), in contrast to Study 1 where both factors explained a similar amount of variance (17\% and 16\%), the first factor for Studies $2-4$ explained $25 \%$ of the variance and the second factor explained $7 \%$.

\section{Studies 2-4 Interim Discussion}

The pre-registered CFA for the two-factor structure uncovered in Study 1 consistently resulted in the best fit indices for the data in Studies 2-4 (Table 1). The combinedsample TLI and CFI values did not reach the pre-registered inference criterion of 0.85 , though Study 1 also failed to meet this criterion. In contrast, the RMSEA did surpass the interference criteria of 0.10 , though this was true of the one-factor models as well as the two-factor model. These mixed results are further complicated by the additional EFA results. While item 20 consistently loaded on no factor in either sample, other item-loadings were very different (see Supplemental Materials). While the fit indices and secondary EFA consistently support a two-factor structure, the low TLI/CFI and inconsistent item-loading in the EFA calls into question the replicability of the factor structure found in Study 1. The following exploratory analyses provide additional contextualization by investigating correlations between various constructs of interest and the total and subscale scores across studies 1-4.

\section{Exploratory Analysis for Construct Validation}

Mean wisdom scores for Studies 1-4 are shown in Figure 3. Given the availability of demographics and other scales in these studies, we report correlations with BWSS Wisdom total-score and Poise and Attunement subscale scores with each of the scales (Table 2). For Study 1 (Table 2a), we predicted that wisdom scores should positively correlate with mood and with the BFI-2-openness subscale; wisdom should negatively correlate with the BFI-2-negative emotionality subscale and dysfunctional attitudes. For Study 2 (Table $2 \mathrm{~b}$ ), we predicted that wisdom should positively correlate with scales indicative of beneficial qualities (TIPIopenness subscale, FFMQ-SF, ERQ-reappraisal subscale, EQ, RRQ-reflection subscale) and negatively correlate with scales indicative of detrimental qualities (TIPI-neuroticism subscale, PHQ-SADS subscales, AAQ-II, ERQ-suppression subscale, RRQ-rumination subscale); other TIPI subscales are reported, though no predictions were made. For Studies 3-4 (Table 2c), wisdom scores should correlate positively with mood measured by the mood-board and PANAS while correlating negatively with the inattentiveness measured by the ARCES and MEWS. Correlations with age, gender, and education are reported where available, though no predictions were made. These correlations are exploratory and should be taken as descriptive, not inferential (hence the lack of statistical tests). They are meant to be indicative of convergent and discriminant validity by estimating effect sizes; we shall await further replications to demonstrate their robustness in novel samples.

Across all studies, predictions were remarkably accurate, including several strong correlations $(|\mathrm{r}|>0.5)$ and some extremely strong correlations $(|\mathrm{r}|>0.7)$. Inaccurate predictions include the lack of correlation with the ERQ subscale for Suppression $(|\mathrm{r}|<0.1)$ and the weaker than expected correlations with the ARCES and MEWS ( $|\mathrm{r}|$ $<0.2$ ). The strongest negative correlations were with the personality trait neuroticism, measured in both Studies 1 and 2 by the BFI- 2 and the TIPI respectively. The strongest positive correlation was with the Experiences Questionnaire, a measure of decentering. 


\section{Preprint}

\section{Table 2}

Exploratory

correlations between

Total Wisdom, Poise,

and Attunement

with other available

questionnaires.

\begin{tabular}{|l|c|c|c|}
\hline Table 2a & Study 1 & & \\
\hline Scale & BWSS & Poise & Attunement \\
\hline BWSS & 1.00 & 0.87 & 0.81 \\
\hline Poise & 0.87 & 1.00 & 0.44 \\
\hline Attunement & 0.81 & 0.44 & 1.00 \\
\hline Age & -0.04 & -0.07 & 0.01 \\
\hline Gender & -0.05 & -0.17 & 0.10 \\
\hline Education & 0.10 & 0.05 & 0.14 \\
\hline Mood Valence & 0.45 & 0.49 & 0.24 \\
\hline Mood Intensity & -0.01 & -0.07 & 0.05 \\
\hline BFI OpenMind & 0.47 & 0.30 & 0.53 \\
\hline BFI AestheticSensitivity & 0.26 & 0.07 & 0.41 \\
\hline BFI IntellectualCuriosity & 0.44 & 0.31 & 0.46 \\
\hline BFI Creativelmagination & 0.38 & 0.33 & 0.33 \\
\hline BFI Negative Emotionality & -0.58 & -0.76 & -0.17 \\
\hline BFI Anxiety & -0.54 & -0.70 & -0.16 \\
\hline BFI Depression & -0.56 & -0.68 & -0.22 \\
\hline BFI EmotionalVolatility & -0.46 & -0.66 & -0.06 \\
\hline DAS & -0.54 & -0.59 & -0.28 \\
\hline DAS Perfectionism & -0.53 & -0.55 & -0.31 \\
\hline DAS Dependency & -0.46 & -0.55 & -0.18 \\
\hline
\end{tabular}




\begin{tabular}{|l|c|c|c|}
\hline Table 2b & Study 2 & & \\
\hline Scale & BWSS & Poise & Attunement \\
\hline BWSS & 1.00 & 0.94 & 0.83 \\
\hline Poise & 0.94 & 1.00 & 0.61 \\
\hline Attunement & 0.83 & 0.61 & 1.00 \\
\hline Age & -0.11 & -0.14 & -0.02 \\
\hline Gender & -0.15 & -0.18 & -0.03 \\
\hline TIPI Openness & 0.42 & 0.33 & 0.41 \\
\hline FFMQ Observe & 0.54 & 0.53 & 0.42 \\
\hline FFMQ Describe & 0.48 & 0.40 & 0.48 \\
\hline FFMQ ActAware & 0.50 & 0.51 & 0.30 \\
\hline FFMQ NJudge & 0.46 & 0.49 & 0.26 \\
\hline FFMQ NReact & 0.65 & 0.72 & 0.37 \\
\hline ERQ Re & 0.50 & 0.50 & 0.39 \\
\hline EQ & 0.80 & 0.81 & 0.57 \\
\hline RRQ Re & 0.46 & 0.34 & 0.45 \\
\hline TIPI Neuroticism & -0.72 & -0.79 & -0.41 \\
\hline PHQ 15 & -0.25 & -0.31 & -0.07 \\
\hline GAD 7 & -0.54 & -0.61 & -0.26 \\
\hline PHQ 9 & -0.50 & -0.54 & -0.31 \\
\hline AAQ & -0.54 & -0.60 & -0.28 \\
\hline ERQ Su & -0.06 & -0.08 & 0.03 \\
\hline RRQ Ru & -0.64 & -0.71 & -0.35 \\
\hline TIPI Extraversion & 0.19 & 0.21 & 0.09 \\
\hline TIPI Agreeableness & 0.25 & 0.20 & 0.21 \\
\hline TIPI Conscientiousness & 0.32 & 0.31 & 0.27 \\
\hline & & & \\
\hline
\end{tabular}

\begin{tabular}{|l|c|c|c|}
\hline Table 2c & Study 3-4 & & \\
\hline Scale & BWSS & Poise & Attunement \\
\hline BWSS & 1.00 & 0.90 & 0.85 \\
\hline Poise & 0.90 & 1.00 & 0.56 \\
\hline Attunement & 0.85 & 0.56 & 1.00 \\
\hline Age & 0.13 & 0.15 & 0.08 \\
\hline Gender & -0.05 & -0.14 & 0.05 \\
\hline Mood Valence & 0.30 & 0.32 & 0.19 \\
\hline Mood Intensity & -0.08 & -0.14 & 0.02 \\
\hline PANAS & 0.37 & 0.36 & 0.27 \\
\hline PANAS Positive & 0.26 & 0.16 & 0.33 \\
\hline ARCES & -0.10 & -0.12 & -0.02 \\
\hline MEWS & -0.17 & -0.24 & 0.00 \\
\hline PANAS Negative & -0.21 & -0.29 & -0.04 \\
\hline
\end{tabular}




\section{Preprint}

\section{Figure 3}

Plots of BWSS scores across studies. Plot width reflects sample size.

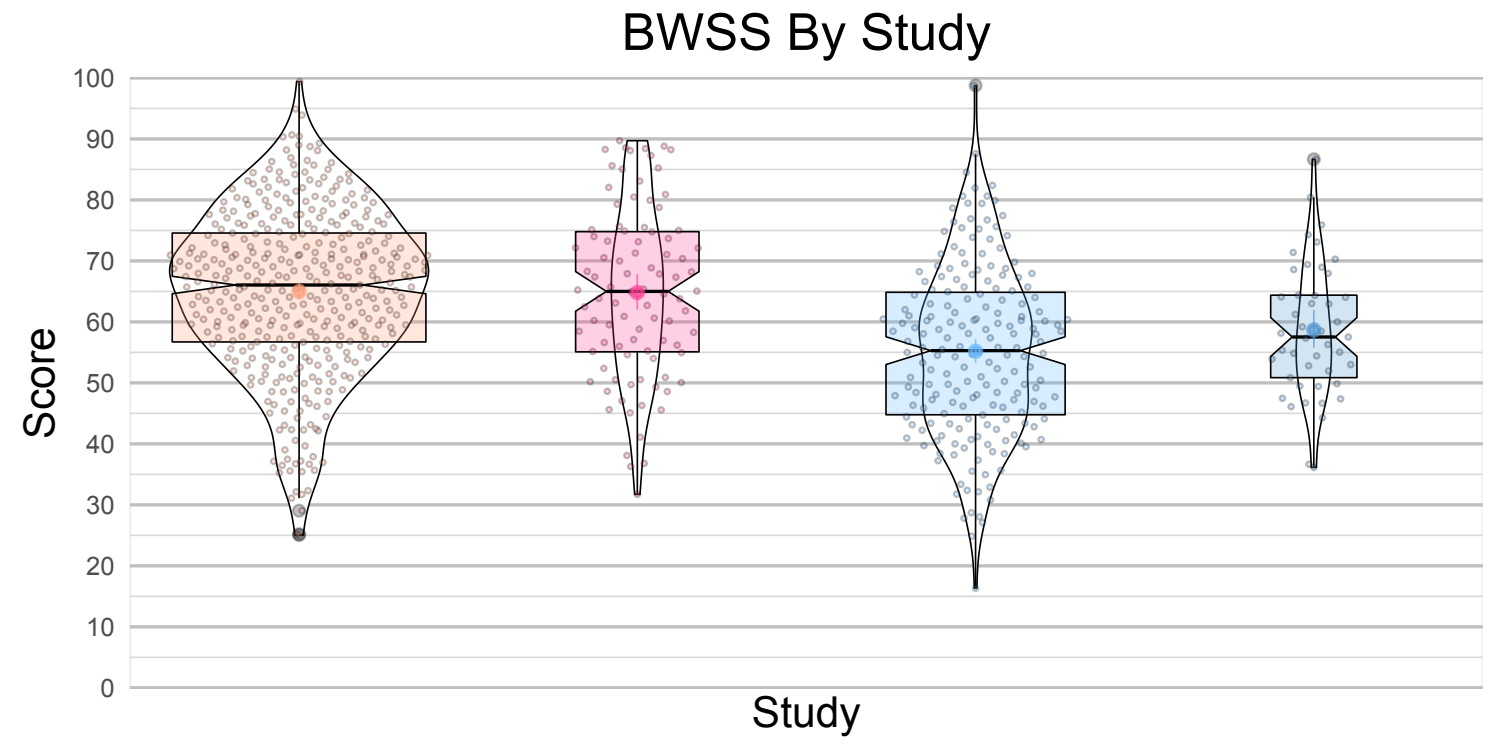

Study $\boxminus \exists$ Microdose Study (1) $\boxminus \exists$ MAP Study (2) $\boxminus \exists$ MCT Long (3) $\boxminus \exists$ MCT B\&W (4)
Concerning the BWSS subscales, the Poise subscale appears more strongly positively correlated with beneficial constructs, particularly those related to non-reactivity, and more negatively correlated with detrimental constructs than the Attunement subscale. The exception to this finding is that Attunement is more positively correlated with trait Openness and with the Reflection subscale of the RRQ. We discuss these findings in greater detail below.

\section{General Discussion}

The original Brief Wisdom Screening Scale (BWSS) was a 21 -item questionnaire for quickly assessing selfreported wisdom. Our exploratory and confirmatory factor analyses of 720 participants across four studies indicates that a two-factor solution for the BWSS is superior to the onefactor model. We propose an update to the measurement of the BWSS such that $0-100$ visual analog scales are preferred to Likert-type scales while also proposing a scoring method that drops one poor-loading item (item 20). The twofactor solution allows for measuring a total Wisdom score alongside two subscales: Poise and Attunement. The BWSS and its subscale correlations were explored, demonstrating excellent convergent and discriminant validity. While "wisdom" is notoriously difficult to define (Baltes et al., 2002), our findings suggest the BWSS serves its purpose as a quick, easy to understand self-report measure and that these two subscales may lend themselves to further study of two important facets of wisdom.

Poise reflects the internal stability of mind seen in wise individuals who are not brought down by the momentto-moment struggles of life. Poise reflects self-integration, mental imperturbability, and emotional stability. Poise reflects the experience of inner peace and stability characteristic of wisdom as seen in the powerful negative correlations with counterproductive internal states and traits such as neuroticism, rumination, psychological inflexibility, emotional volatility, anxiety, and depression. Correlations also show a lack of endorsement of dysfunctional attitudes that hamper the enjoyment of life. Reinforcing this point, Poise strongly positively correlated with mindful nonreactivity and "decentering": an especially psychologically healthful perspective wherein thoughts and emotions are accepted from a detached state of mind, the lack of which is associated with psychosocial dysfunction (Fresco et al., 2007).

Attunement reflects the propensity to be changed by and learn from experience. Attunement showed stronger correlations with trait open-mindedness and openness, as well as with the propensity to reflect (rather than ruminate) on experience. Overall, most of the correlations with Attunement were in the same direction as with Poise, but with lower absolute magnitude. Given the various questionnaires assessed across the four studies, this may reflect the fact that none of them specifically address the interpersonal and experiential learning over time implied by Attunement. Indeed, the particular appetite for learning from life's experiences may reflect part of what makes wisdom so hard to define. What does it mean to "learn from life" when we all have such different experiences? Learning from life must involve a process of abstraction from the finite, concrete, primary experience of the individual to a broader principle or insight about a shared human condition. Attunement may reflect an orientation toward life, a tendency toward distillation, akin to the "metaheuristic" perspective of wisdom discussed by Baltes \& Staudinger (2000). Indeed, wisdom can be conceived as "expertise in the conduct of life", i.e. practical and procedural knowledge about the fundamental pragmatics of life (Baltes et al., 2002). To obtain such expertise, merely living is not enough (Baltes et al., 2002); to gain wisdom, one must learn from experiences, distilling useful lessons from one situation so they may be fruitfully applied to novel situations without losing track of relevant contextual caveats. This optimization-orientation toward learning from 
life surely deserves additional study.

To confirm the superiority of this two-factor solution, which was uncovered using a community sample mostly composed of psychedelic microdosers, we collected new samples and pre-registered a confirmatory factor analysis. Low recruitment in the registered sample of meditators led to collection of two additional samples of undergraduate students. Our findings (Table 1) supported the registered CFA showing that the two-factor solution was superior. Exploratory analyses also replicated that one item poorly loads on both factors; as such, we recommend not including this item in future scoring of the BWSS. The exploratory EFA, however, revealed that the different samples resulted in different proposed item loadings and factors explaining different proportions of variance, thus it is too soon to conclude that the specific Poise-Attunement factor structure found in Study 1 is detectable across samples with different background characteristics. Indeed, it may be the case that response characteristics change depending on the group under study: the community sample of microdosers may differ on other personality and lifestyle characteristics compared to the international sample of meditators, each of which would be expected to differ from the locally available undergraduate sample.

\section{Constraints on Generality}

As mentioned above, the EFA for Studies 2-4 did not result in identical item loadings as that for Study 1; as such, we cannot conclusively say that this particular factor structure will replicate across samples with different characteristics. Participants in Study 1 were primarily psychedelics-experienced adults (80\% male) whereas Study 2 were adult meditators ( $40 \%$ male) and Studies 3-4 were young-adult undergraduates with diverse ethnic and religious backgrounds (27\% male). Samples taken from homogenous groups with similar underlying characteristics may continue to result in different item-loadings if the BWSS is again subjected to EFA; what appears consistent is that a two-factor structure fits the data better than a onefactor structure. Future research will be needed to inform whether the Poise-Attunement subscales identified here are differently responsive to intervention and continue to be associated with different personality traits and individual differences. Total Wisdom, Poise, and Attunement scores may interact with psychopathology; fully half of Study 1 reported some mental health diagnosis (Rosenbaum et al., 2020) whereas this measure was not available in Studies $2-4$. On the other hand, meditation training may plausibly result in changes to wisdom-related response profiles. We have no reason to believe that the results depend on other characteristics of the participants, materials, or context. This Constraints on Generality (COG) statement is made in accordance with Simmons et al. (Simons et al., 2017).

\section{Future Directions}

Future research is needed to investigate the extent to which Poise and Attunement are responsive to interventions. Indeed, Study 1 used the BWSS in the interest of measuring differences in wisdom between self-reported psychedelic microdosers and their non-microdosing peers (Anderson, Petranker, Rosenbaum, et al., 2019). Planned randomized placebo-controlled trials on microdosing will incorporate this measure of wisdom in order to test whether microdosing can increase wisdom, as has been qualitatively reported in cross-sectional research (Anderson, Petranker, Christopher, et al., 2019).

Clinical research may also find that wisdom could be a target of intervention and may attempt to intervene on Poise and Attunement in different ways. For example, one may find it difficult to reflect during or following life's challenging experiences if one lacks the Poise to prevent being drawn into rumination and despair. Future intervention research could specifically target Poise for enhancement, e.g. by equanimity-focused meditation training or decentering-focused cognitive-behavioural therapy, and consider whether increasing Poise could indirectly increase Attunement. A competing theoretical strategy could be to promote an Attunement orientation by highlighting the utility of reflection on experience or by providing skill training and activities meant to facilitate the distillation and learning process characteristic of Attunement. Such an intervention could test whether increasing Attunement could indirectly increase Poise. Each of these questions could be studied with a longitudinal design using time-lagged statistical modelling to inform empirically focused wisdom training and theory.

In addition to the aforementioned intervention research aimed at enhancing wisdom by part or as a whole, additional measure validation work that combines different measures of wisdom is also warranted. One important factor in any self-report measure is ensuring that the measure is not rendered obsolete by overly biased self-perceptions. As such, the BWSS could be combined with informant reports one wisdom and could be measured alongside the more extensive task-based and interview measures of wisdom. Indeed, there still remains space for development of exciting novel measures of advice-based assessments of wisdom incorporating video-vignettes rather than written stories (Glück, 2018). One wonders whether the future of wisdom measurement could even incorporate virtual reality scenarios with video-game logic to progress through challenging scenes.

\section{Conclusion}

There lies in each one of us a wellspring of sad memories and negative emotion readily available for those who wish to drown in sorrow yet, simultaneously, each of us holds within ourselves the capacity for the greatest human happiness. Life is a complex game, the navigation of which is an underdetermined optimization problem. Wisdom, conceived as "expertise in the conduct of life", can be understood as a "metaheuristic" intended to more optimally traverse life's opportunity landscape. While the exact definitions of wisdom are still being debated, various tools exist to operationalize wisdom, one of which is the empirically constructed BWSS. The two empirically derived factors, Poise and Attunement, may further serve empirical investigations into this topic, especially under pragmatic constraints where longer task-based measures of wisdom are not feasible. More research is needed, but wisdom promises to remain an interesting and fruitful area of human study for quite some time. 


\section{Data Availability Statement}

The survey materials, data, and analysis code for all experiments are available on the Open Science Framework (Anderson, 2018a). Study 2 was preregistered (Anderson, 2018b); deviations from the preregistration are described in the methods section. To facilitate peer-review, we have created a view-only link for our OSF project, which will be made public upon acceptance: https://osf.io/szhft/?view_ only $=0 \mathrm{~d} 2 \mathrm{ed} 0 \mathrm{~b} 3928 \mathrm{~d} 4 \mathrm{f} 2599 \mathrm{c} 2 \mathrm{f} 8 \mathrm{f} 7336 \mathrm{~b} 9 \mathrm{a} 28$

\section{Funding}

This work was supported by the Canada Graduate Doctoral Scholarships program the Natural Sciences and Engineering Research Council of Canada (NSERC) [grant number PGSD2 - 518591], the Oxford Mindfulness Trust, and a Mind and Life 1440 Award ("Does ongoing practice of mindfulness meditation promote healthy relationships?”).

\section{References}

Anderson, T. (2018a). Confirmatory Factor Analysis of Brief Wisdom Screening Scale. https://osf.io/szhft/

Anderson, T. (2018b). Preregistration of Confirmatory Factor Analysis of Brief Wisdom Screening Scale. https:// osf.io/d7495

Anderson, T., Petranker, R., Christopher, A., Rosenbaum, D., Weissman, C., Dinh-Williams, L.-A., Hui, K., \& Hapke, E. (2019). Psychedelic microdosing benefits and challenges: An empirical codebook. Harm Reduction Journal, 16(1), 43. https://doi.org/10.1186/s12954-0190308-4

Anderson, T., Petranker, R., Rosenbaum, D., Weissman, C. R., Dinh-Williams, L.-A., Hui, K., Hapke, E., \& Farb, N. A. S. (2019). Microdosing psychedelics: Personality, mental health, and creativity differences in microdosers. Psychopharmacology, 236(2), 731-740. https://doi.org/10.1007/s00213-018-5106-2

Baltes, P. B., Glück, J., \& Kunzmann, U. (2002). Wisdom: Its structure and function in regulating successful life span development. In Handbook of positive psychology. (pp. 327-347). Oxford University Press.

Baltes, P. B., \& Staudinger, U. M. (2000). Wisdom: A metaheuristic (pragmatic) to orchestrate mind and virtue toward excellence. American Psychologist, 55(1), 122-136. https://doi.org/10.1037/0003-066X.55.1.122

Bohlmeijer, E., ten Klooster, P. M., Fledderus, M., Veehof, M., \& Baer, R. (2011). Psychometric Properties of the Five Facet Mindfulness Questionnaire in Depressed Adults and Development of a Short Form. Assessment, 18(3), 308-320. https://doi. org/10.1177/1073191111408231

Bond, F. W., Hayes, S. C., Baer, R. A., Carpenter, K. M., Guenole, N., Orcutt, H. K., Waltz, T., \& Zettle, R. D. (2011). Preliminary Psychometric Properties of the Acceptance and Action Questionnaire-II: A Revised Measure of Psychological Inflexibility and Experiential Avoidance. Behavior Therapy, 42(4), 676-688. https://doi. org/10.1016/j.beth.2011.03.007

Cheyne, J. A., Carriere, J. S. A., \& Smilek, D. (2006). Absent-mindedness: Lapses of conscious awareness and everyday cognitive failures. Consciousness and
Cognition, 15(3), 578-592. https://doi.org/10.1016/j. concog.2005.11.009

de Graaf, L. E., Roelofs, J., \& Huibers, M. J. H. (2009). Measuring Dysfunctional Attitudes in the General Population: The Dysfunctional Attitude Scale (form A) Revised. Cognitive Therapy and Research, 33(4), 345-355. https://doi.org/10.1007/s10608-009-9229-y

Epskamp, S., Stuber, S., Nak, J., Veenman, M., \& Jorgensen, T. D. (2019). semPlot: Path Diagrams and Visual Analysis of Various SEM Packages' Output (Version 1.1.2) [Computer software]. https://CRAN.R-project.org/ package $=$ semPlot

Farb, N., Anderson, T., Segal, Z. V., \& Hood, P. (2018, February 8). Contemplative Practice, Well Being, and Social Function. OSF. https://osf.io/w2f86/

Fresco, D. M., Moore, M. T., van Dulmen, M. H. M., Segal, Z. V., Ma, S. H., Teasdale, J. D., \& Williams, J. M. G. (2007). Initial Psychometric Properties of the Experiences Questionnaire: Validation of a Self-Report Measure of Decentering. Behavior Therapy, 38(3), 234 246. https://doi.org/10.1016/j.beth.2006.08.003

Glück, J. (2018). Measuring Wisdom: Existing Approaches, Continuing Challenges, and New Developments. The Journals of Gerontology: Series B, 73(8), 1393-1403. https://doi.org/10.1093/geronb/gbx140

Glück, J., König, S., Naschenweng, K., Redzanowski, U., Dorner, L., Straßer, I., \& Wiedermann, W. (2013). How to measure wisdom: Content, reliability, and validity of five measures. Frontiers in Psychology, 4. https://doi. org/10.3389/fpsyg.2013.00405

Gosling, S. D., Rentfrow, P. J., \& Swann, W. B. (2003). A very brief measure of the Big-Five personality domains. Journal of Research in Personality, 37(6), 504 528. https://doi.org/10.1016/S0092-6566(03)00046-1

Gross, J. J., \& John, O. P. (2003). Individual differences in two emotion regulation processes: Implications for affect, relationships, and well-being. Journal of Personality and Social Psychology, 85(2), 348-362. https://doi.org/10.1037/0022-3514.85.2.348

Igor Grossmann, Nic M. Weststrate, Monika Ardelt, Justin P. Brienza, Mengxi Dong, Michel Ferrari, Marc A. Fournier, Chao S. Hu, Howard C. Nusbaum, \& John Vervaeke. (submitted). The Science of Wisdom in a Polarized World: Knowns and Unknowns. Psychological Inquiry.

Kroenke, K., Spitzer, R. L., Williams, J. B. W., \& Löwe, B. (2010). The Patient Health Questionnaire Somatic, Anxiety, and Depressive Symptom Scales: A systematic review. General Hospital Psychiatry, 32(4), 345359. https://doi.org/10.1016/j.genhosppsych.2010.03.006

Mowlem, F. D., Skirrow, C., Reid, P., Maltezos, S., Nijjar, S. K., Merwood, A., Barker, E., Cooper, R., Kuntsi, J., \& Asherson, P. (2019). Validation of the Mind Excessively Wandering Scale and the Relationship of Mind Wandering to Impairment in Adult ADHD. Journal of Attention Disorders, 23(6), 624-634. https://doi. org/10.1177/1087054716651927

R Core Team. (2014). R: A Language and Environment for Statistical Computing. R Foundation for Statistical Computing. http://www.r-project.org/

Revelle, W. (2020). psych: Procedures for 
Psychological, Psychometric, and Personality Research (Version 1.9.12.31) [Computer software]. https://CRAN.R-

project.org/package $=$ psych

Rosenbaum, D., Weissman, C., Anderson, T., Petranker, R., Dinh-Williams, L.-A., Hui, K., \& Hapke, E. (2020). Microdosing psychedelics: Demographics, practices, and psychiatric comorbidities. Journal of Psychopharmacology, 0269881120908004 . https://doi. org/10.1177/0269881120908004

Rosseel, Y., Oberski, D., Byrnes, J., Vanbrabant, L., Savalei, V., Merkle, E., Hallquist, M., Rhemtulla, M., Katsikatsou, M., Barendse, M., Chow, M., \& Jorgensen, T. D. (2018). lavaan: Latent Variable Analysis (Version 0.6-3) [Computer software]. https://CRAN.R-project.org/ package=lavaan

Simons, D. J., Shoda, Y., \& Lindsay, D. S.

(2017). Constraints on Generality (COG): A Proposed Addition to All Empirical Papers. Perspectives on Psychological Science, 12(6), 1123-1128. https://doi. org/10.1177/1745691617708630

Soto, C. J., \& John, O. P. (2017). The next Big Five Inventory (BFI-2): Developing and assessing a hierarchical model with 15 facets to enhance bandwidth, fidelity, and predictive power. Journal of Personality and Social Psychology, 113(1), 117-143. https://doi.org/10.1037/ pspp0000096

Trapnell, P. D., \& Campbell, J. D. (1999). Private self-consciousness and the five-factor model of personality: Distinguishing rumination from reflection. Journal of Personality and Social Psychology, 76(2), 284-304. https:// doi.org/10.1037/0022-3514.76.2.284

Watson, D., Clark, L. A., \& Tellegen, A. (1988).

Development and validation of brief measures of positive and negative affect: The PANAS scales. Journal of Personality and Social Psychology, 54(6), 1063-1070. https://doi.org/10.1037/0022-3514.54.6.1063

Wickham, H. (2016). ggplot2: Elegant Graphics for Data Analysis. Springer-Verlag New York. http://ggplot2.org

Wisdom, n. (n.d.). In OED Online. Oxford University Press. Retrieved 20 February 2020, from https://www.oed. com/view/Entry/229491 


\section{Appendix - Factor Loadings for Study 1}

\begin{tabular}{|c|c|c|}
\hline Item Text & Poise & Attunement \\
\hline $\begin{array}{l}\text { [14] I either get very angry or depressed if things go } \\
\text { wrong. }\end{array}$ & 0.83 & -0.20 \\
\hline [06] My peace of mind is not easily upset. & 0.77 & -0.01 \\
\hline $\begin{array}{l}\text { [10] Sometimes I get so charged up emotionally that I am } \\
\text { unable to consider all ways of dealing with my problems. }\end{array}$ & 0.80 & -0.29 \\
\hline $\begin{array}{l}\text { [09] At this point in my life, I find it easy to laugh at my } \\
\text { mistakes. }\end{array}$ & 0.55 & 0.20 \\
\hline $\begin{array}{l}\text { [04] I can freely express my emotions without feeling like I } \\
\text { might lose control. }\end{array}$ & 0.46 & 0.18 \\
\hline $\begin{array}{l}\text { [11] My happiness is not dependent on other people and } \\
\text { things. }\end{array}$ & 0.43 & 0.16 \\
\hline [13] I am able to integrate the different aspects of my life. & 0.39 & 0.35 \\
\hline [01] I don't worry about other people's opinions of me. & 0.40 & 0.09 \\
\hline [05] I can accept the impermanence of things. & 0.35 & 0.28 \\
\hline [03] I have a good sense of humor about myself. & 0.31 & 0.32 \\
\hline [17] I always try to look at all sides of a problem. & 0.24 & 0.40 \\
\hline [20] There are some people I know I would never like. & 0.12 & -0.08 \\
\hline [19] I am tuned in to my own emotions. & 0.01 & 0.61 \\
\hline $\begin{array}{l}\text { [15] I like to read books which challenge me to think } \\
\text { differently about issues. }\end{array}$ & 0.00 & 0.39 \\
\hline [18] I often have a sense of oneness with nature. & -0.04 & 0.57 \\
\hline [08] I've learned valuable life lessons from others. & -0.09 & 0.65 \\
\hline $\begin{array}{l}\text { [12] I'm very curious about other religious and/or } \\
\text { philosophical belief systems. }\end{array}$ & -0.07 & 0.41 \\
\hline $\begin{array}{l}\text { [02] I have dealt with a great many different kinds of } \\
\text { people during my lifetime. }\end{array}$ & -0.11 & 0.63 \\
\hline $\begin{array}{l}\text { [21] It seems I have a talent for reading other people's } \\
\text { emotions. }\end{array}$ & -0.11 & 0.57 \\
\hline [07] I have grown as a result of losses I have suffered. & -0.15 & 0.66 \\
\hline $\begin{array}{l}\text { [16] I feel that my individual life is a part of a greater } \\
\text { whole. }\end{array}$ & -0.19 & 0.55 \\
\hline \multirow[t]{2}{*}{ Factor Correlations: } & 0.54 & \\
\hline & Poise & Attunement \\
\hline SS Loadings & 3.26 & 3.53 \\
\hline Proportion Variance & 0.17 & 0.16 \\
\hline Multiple R square of scores with factors & 0.87 & 0.87 \\
\hline
\end{tabular}




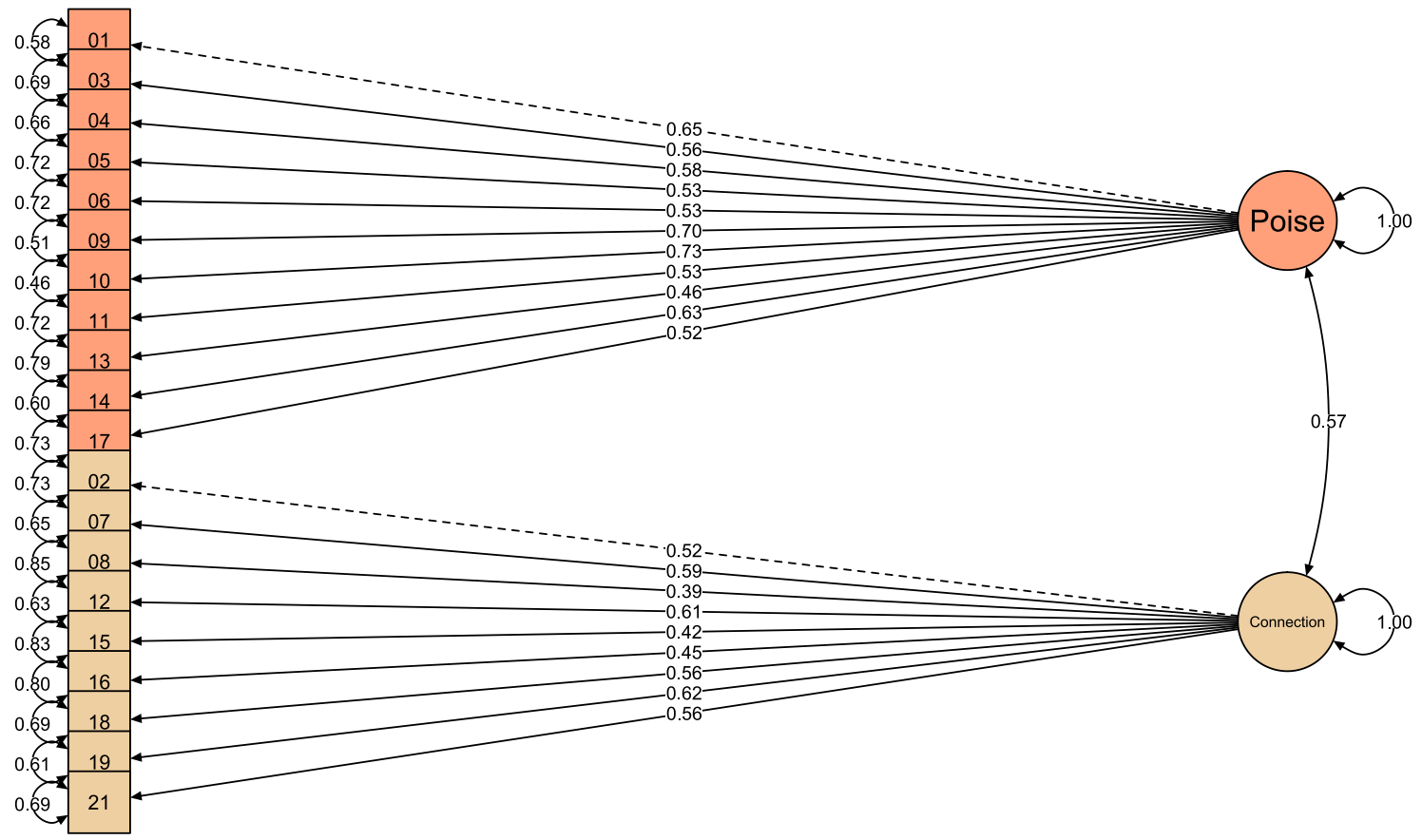

Figure 4

Plot of two-factor structure in Study 1. Coefficients are standardized.

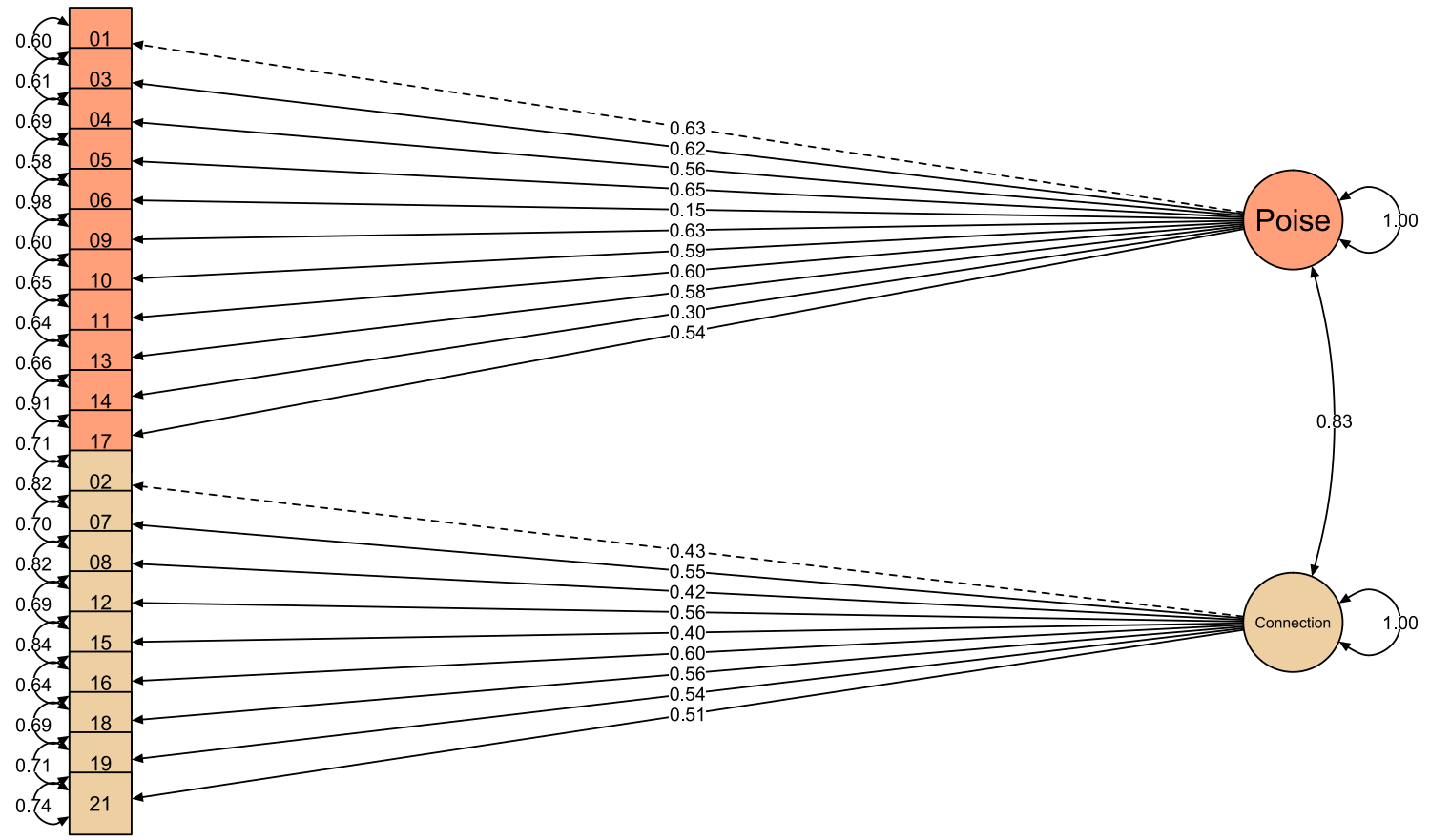

Figure 5

Plot of two-factor structure in Studies 2-4. Coefficients are standardized. 